\title{
ON ALGEBRAIC VARIETIES WHOSE UNIVERSAL COVERING MANIFOLDS ARE COMPLEX AFFINE 3-SPACES
}

\author{
BY SHIGERU IITAKA ${ }^{1}$
}

Communicated by M. F. Atiyah, March 23, 1972

1. Introduction. Let $V$ be a nonsingular projective algebraic variety defined over the field of complex numbers. By $\tilde{V}$ we denote the universal covering manifold of $V$. It is clear that if $\tilde{V}$ is an abelian variety, then $\tilde{V}$ turns out to be a complex affine space. The author is concerned with a converse of this fact. Thus, he proposes the following:

CoNJECTURE $U_{n}$. Suppose that $\tilde{V}$ is a complex affine $n$-space. Then there exists an abelian variety which is a finite unramified covering manifold of $V$.

This has been solved only for $n=1,2$. We note that the proof for $n=2$ requires a detailed study of the classification of algebraic surfaces. In his thesis [3], the author introduced the notion of Kodaira dimension $\kappa(V)$ of algebraic varieties $V$ and by using it he intends to extend the classification theory into higher dimensional case (see [5]). In this note, he shall give a sketchy proof of the following partial solution of $U_{3}$.

THEOREM. Suppose that $V$ satisfies the hypothesis for $U_{3}$. Then $\kappa(V) \neq 1$ and 3.

The detailed proof and related results will appear elsewhere.

2. Divisor-dimension and Kodaira dimension. We recall definitions and some results concerning divisor-dimension and Kodaira dimension (see [3]). Let $V$ be a complete algebraic variety and $D$ a Cartier divisor on $V$. Denoting by $\mathcal{O}(D)$ the invertible sheaf associated with $D$, we define $l(D)$ to be $\operatorname{dim} \Gamma\left(V^{*}, \mu^{*} \mathcal{O}(D)\right)$ where $\mu: V^{*} \rightarrow V$ is a normalization of $V$. We study $l(m D)$ as a function of $m$ for sufficiently large integer $m$. If there exists a positive integer $m_{0}$ such that $l\left(m_{0} D\right)>0$, we can find real positive constants $\alpha, \beta$ and a nonnegative integer $\kappa$ which satisfy

$$
\alpha m^{\kappa} \leqq l\left(m \cdot m_{0} D\right) \leqq \beta m^{\kappa}
$$

for sufficiently large values of $m$. Since the $\kappa$ is independent of the choice

AMS 1970 subject classifications. Primary 14J15; Secondary 14E30, 14K22, 32J15.

Key words and phrases. Classifications of algebraic varieties.

${ }^{1}$ Supported in part by National Science Foundation grant GP-7952X3. 
of $\alpha, \beta$, and $m_{0}$, we define $D$-dimension of $V$, written $\kappa(D, V)$, to be the $\kappa$. If $l(m D)=0$ for any $m \geqq 1$, we set $\kappa(D, V)=-\infty$. Clearly, we have $\kappa(D, V)=\kappa(r D, V)$ for any $r \geqq 1$. Hence, we can define $\kappa(D, V)$ for a fractional divisor $D$. Now let $V$ be an algebraic variety. By Hironaka, there exists a nonsingular projective model $V^{*}$ of $V$. We indicate by $K\left(V^{*}\right)$ a canonical divisor of $V^{*}$. Define the $m$-genus $P_{m}(V)$ to be $l\left(m K\left(V^{*}\right)\right)$ and also define the Kodaira dimension $\kappa(V)$ to be $\kappa\left(K\left(V^{*}\right), V^{*}\right)$. Suppose that $\kappa(V) \geqq 0$. Then we can find a fiber space $f: V^{*} \rightarrow W, V^{*}, W$ being nonsingular projective algebraic varieties with the following properties:

(i) $V^{*}$ is birationally equivalent to $V$,

(ii) $\operatorname{dim} W=\kappa(V)$,

(iii) any general fiber $V_{w}^{*}$ is irreducible;

(iv) $\kappa\left(V_{w}^{*}\right)=0$.

Moreover, these properties characterize $f: V^{*} \rightarrow W$ up to birational equivalence. Hence, we call $f: V^{*} \rightarrow W$ a canonical fiber space associated to $V$.

3. A proof of $U_{2}$. First, we notice some basic properties which $V$ has, if $V$ satisfies the hypothesis for $U_{n}$.

Proposition 1. Let $W$ be a subvariety of $V$ and $W^{*}$ a nonsingular model of $W$. Then the fundamental group of $W^{*}$ is infinite.

Hence, there are no rational curves on $V$. This implies that $V$ is strongly minimal (for the definition, see [9]).

Proposition 2 (Kodaira). $\kappa(V)<n$.

For the proof, we refer to [6].

By using these, we shall sketch the proof of $U_{2}$.

Case I. $\kappa(V)=1$. In this case, we shall derive a contradiction in the following five steps. $(\alpha)$ By a theorem due to the Italian school, we see the existence of an elliptic fiber space $f: V \rightarrow W$. That is to say, $V$ is an elliptic surface. $(\beta)$ Any singular fiber of an elliptic surface consists of rational curves or is a multiple of an elliptic curve (see the table of singular fibers in [7]). Hence, the singular fibers $f^{*}\left(a_{1}\right), \ldots, f^{*}\left(a_{s}\right)$ of the elliptic surface $V$ are multiples of elliptic curves $f^{-1}\left(a_{1}\right), \ldots, f^{-1}\left(a_{s}\right)$, respectively. Thus, we have $f^{*}\left(a_{1}\right)=e_{1} f^{-1}\left(a_{1}\right), \ldots, f^{*}\left(a_{s}\right)=e_{s} f^{-1}\left(a_{s}\right)$. $(\gamma)$ The canonical bundle formula (see [8], [2]) reads

$$
k(V)=\kappa\left(K(W)+\sum\left(1-1 / e_{j}\right) a_{j}, W\right) .
$$

Therefore, $2 \pi-2+\sum\left(1-1 / e_{j}\right)>0$ follows, where $\pi$ is the genus of $W$. $(\delta)$ We can construct the universal covering manifold $W^{*}$ which ramifies 
at every point over each $a_{j}$ with the multiplicity $e_{j}$ for any $1 \leqq j \leqq s$. Then, $V_{1}=V \times{ }_{W} \tilde{W}^{*}$ is an unramified covering manifold of $V$. As a consequence, $(\varepsilon)$ we obtain a surjective holomorphic mapping from $C^{2}=\tilde{V}_{1}$ onto $\tilde{W}^{*}$, a complex upper half plane. On the other hand, in view of the Liouville theorem, we see that $f$ is constant.

Case II. $\kappa(V)=0$. By the classification theory of algebraic surfaces, if $\pi_{1}(V)$ is infinite, then $V$ is an abelian variety or a hyperelliptic surface which has an abelian variety as a finite unramified covering manifold.

Case III. $\kappa(V)=-\infty$. In this case, from the Enriques criterion we deduce immediately that $V$ is a ruled surface. Therefore, $V$ has many rational curves. This contradicts Proposition 1.

4. The existence of minimal canonical fiber space. In this section, we shall state some analogues for the steps $(\alpha)$ and $(\beta)$ in Case I.

Proposition 3. Let $V$ be a minimal algebraic variety of dimension 3 . Suppose that $\kappa(V)=1$. Then there exists a canonical fiber space $f: V \rightarrow W$ whose general fiber is a minimal surface.

Proposition 4. Under the same assumption as above, we further assume that $V$ is strongly minimal. Then every singular fiber of the fiber space $f: V^{*} \rightarrow W$ has only one irreducible component. A singularity of the irreducible component is negligible. Moreover, if a general fiber is an abelian variety or a hyperelliptic surface, singular fibers are multiples of nonsingular surfaces.

Proposition 5. Let $V$ be a strongly minimal algebraic variety of dimension 3. Suppose that $\kappa(V)=2$. Then there exists a canonical fiber space $f: V \rightarrow W$ such that $W$ is relatively minimal and such that every fiber is (possibly a multiple of) an elliptic curve.

We call the fiber space, constructed in Proposition 3, the minimal canonical fiber space associated with $V$.

In the proofs of these propositions, the following lemmas are useful.

LEMMA 1. Let $f: V \rightarrow W$ be a fiber space of nonsingular projective algebraic varieties such that $\operatorname{dim} W=1$ and $\kappa(V) \geqq 0$. Suppose that a pluri-canonical divisor of a general fiber is linearly equivalent to zero. Then some irreducible component $C_{v}$ of any reducible fiber $f^{*}(a)=\sum_{v=1}^{s} n_{v} C_{v}, s \geqq 2$, has the Kodaira dimension $-\infty$.

LEMMA 2. Let $f: V \rightarrow W$ be an elliptic fiber space of nonsingular projective algebraic varieties such that $\operatorname{dim} V=3$ and $\operatorname{dim} W=2$. Then a surface contained in a fiber is a rational surface. 
5. The canonical bundle formulas and the proof of $k(V) \neq 1$. Let $V$ satisfy the hypothesis for $U_{3}$. Besides, we assume $\kappa(V)=1$. Then the minimal canonical fiber space has an abelian variety or a hyperelliptic surface as its general fiber. Hence we can easily prove the canonical bundle formula:

$$
\kappa(V)=\kappa\left(K(W)+\sum\left(1-1 / e_{j}\right) a_{j}, W\right)
$$

Thus by the assumption we obtain $2 \pi-2+\sum\left(1-1 / e_{j}\right)>0$. Here the notation is the same as in step $(\gamma)$ in Case I. Following the argument in the steps $(\delta)$ and $(\varepsilon)$, we can easily derive a contradiction.

In the case when $\kappa(V)=2$, there is a canonical fiber space $f: V^{*} \rightarrow W$ which has the following property: There exist nonsingular curves $\Delta_{1}, \ldots$, $\Delta_{s}$ on $W$ which satisfy:

(1) for any $w \in W-\bigcup \Delta_{i}$, the fiber $f^{*}(w)$ is regular and for any $a_{i} \in \Delta_{i}$, the fiber $f^{*}\left(a_{i}\right)$ is an $e_{i}$-tuple of an elliptic curve;

(2) $\Delta_{i} \cap \Delta_{j}=\varnothing$ for any $i \neq j$.

The following canonical bundle formula is established:

$$
\kappa(V)=\kappa\left(K(W)+\sum\left(1-1 / e_{j}\right) \Delta_{j}, W\right) .
$$

\section{BIBLIOGRAPHY}

1. J. A. Carlson and P. A. Griffiths, A defect relation for equi-dimensional holomorphic mappings hetween algebraic varieties (to appear).

2. S. Iiıdka, Deformations of compact complex surfaces. II, J. Math. Soc. Japan 22 (1970), 247-261. MR 41 \#6252.

3. — On D-dimensions of algebraic varieties, J. Math. Soc. Japan 23 (1971), 356-373.

4. - On some new birational invariants of algebraic varieties, J. Math. Soc. Japan 24 (1972) (to appear).

5. - Classifications of algebraic varieties, Study Math. Statist. (Dankook University) 4 (1971), 1-19, to be continued (Korean).

6. S. Kobayashi and T. Ochiai, Mappings into compact complex manifolds with negative first Chern class, J. Math. Soc. Japan 23 (1971), 137-148.

7. K. Kodaira, On compact complex analytic surfaces. II, Ann. of Math. (2) 77 (1963), 563-626. MR 32 \#1730.

8. - On the structure of compact analytic surfaces. I, Amer. J. Math. 86 (1964), 751-798. MR 32 \# 4708.

9. $O$. Zariski, Introduction to the problem of minimal models in the theory of algebraic surfaces, Publ. Math. Soc. Japan, no. 4, Math. Soc. Japan, Tokyo, 1958. MR 20 \# 3872.

Department of Mathematics, Faculty of Science, University of Tokyo, Hongo, BUNKYO, TOKYO, JAPAN

School of Mathematics, Institute for Advanced Study, Princeton, New Jersey 08540 (For the academic year 1971/72.) 